\title{
BADLY APPROXIMABLE FUNCTIONS AND INTERPOLATION BY BLASCHKE PRODUCTS
}

\author{
by L. A. RUBEL $\uparrow$ and A. L. SHIELDS \\ (Received 20th January 1975)
}

A continuous function $\phi$ on the unit circle is called badly approximable if $\|\phi-p\|_{\infty} \geqq\|\phi\|_{\infty}$ for all polynomials $p$, where \|\|$_{\infty}$ is the essential supremum norm. In (4), Poreda asked whether every continuous $\phi$ may be written $\phi=\phi_{W}+\phi_{B}$, where $\phi_{W}$ is the uniform limit of polynomials (i.e. $\phi_{W}$ belongs to the disc algebra $A$ ) and $\phi_{B}$ is badly approximable. We call such a function $\phi$ decomposable. In (4), he characterised the badly approximable functions as those of constant non-zero modulus and negative winding number around the origin, i.e. ind $(\phi)<0$. (See (3) for two new proofs of this result.) We show that the answer to Poreda's question is $n o$ in general, but give a necessary and sufficient condition for a given $\phi$ to have such a decomposition. Then we apply this criterion to solve an interpolation problem.

Definition. For $\phi \in C(|z|=1), \phi^{\#}$ is the metric projection of $\phi$ into $H^{\infty}$. That is, $\phi^{\#} \in H^{\infty}$ and

$$
\left\|\phi-\phi^{\#}\right\|_{\infty} \leqq\|\phi-g\|_{\infty} \quad \text { for all } g \in H^{\infty} .
$$

It is well known and easy to prove that there is a unique function $\phi^{\#}$ satisfying this requirement.

Lemma. (Sarason (5)). If $\phi$ is a continuous function on $|z|=1$ then $d\left(\phi, H^{\infty}\right)=d(\phi, A)$.

Theorem. A continuous function $\phi$ is decomposable if and only if $\phi^{*} \in A$.

The proof is immediate once one remarks that the Lemma implies that $\phi$ is badly approximable if and only if $\phi$ is badly $H^{\infty}$-approximable, i.e.

for all $f \in H^{\infty}$.

$$
\|\phi-f\|_{\infty} \geqq\|\phi\|_{\infty}
$$

Since there exists a continuous $\phi$ with $\phi^{\#} \notin A$ (see (1)), it follows that not every $\phi$ is decomposable.

We now apply our Theorem to prove an analogue of the following classical result.

$\dagger$ This research was partially supported by grants from the National Science Foundation. 
Theorem. (Carathéodory.) If $a_{0}, a_{1}, \ldots, a_{n}$ are complex numbers, and if the interpolation problem

$$
F^{(j)}(0)=a_{j}, \quad j=0,1, \ldots, n
$$

has a solution $F$ that is bounded and analytic in the unit disc (i.e. $F \in H^{\infty}$ ) satisfying $|F| \leqq 1$, then there is a Blaschke product of order $\leqq n+1$ that satisfies (1).

Our result has the following statement:

Theorem. The interpolation problem (1) always has a solution of the form $F=\lambda B$, where $\lambda$ is a complex constant, and $B$ is a Blaschke product of order $\leqq n$.

Proof. Assume that the $a_{j}$ are not all zero. Define

$$
f(z)=a_{0}+a_{1} z+\frac{a_{2}}{2 !} z^{2}+\ldots+\frac{a_{n}}{n !} z^{n} .
$$

Then $\phi(z)=f(z) / z^{n+1}$ is a continuous function on $\{|z|=1\}$ that does not belong to the disc algebra $A$. But since $\phi$ satisfies a Lipschitz condition, it surely satisfies Dini's condition $\left(\int_{0+} \omega(t, \phi) / t d t<\infty\right)$ and so by the theorem of Carleson and Jacobs (2) the metric projection $\phi^{\#}$ of $\phi$ into $H^{\infty}$ must belong to $A$. By our Theorem then, we may write

$$
z^{-(n+1)} f-g=\Psi
$$

where $g \in A$ and $\Psi$ is badly approximable. By Poreda's theorem, we may take $\Psi=c \psi$ where $c \neq 0, \Psi$ is unimodular, and ind $(\psi)<0$. But then $z^{n+1} \psi=B$ belongs to $A$. Further, $B$ is unimodular, and ind $(B)=(n+1)-$ ind $(\phi) \leqq n$. Hence $B$ is a Blaschke product of degree $\leqq n$, and the result is proved.

A quite analogous argument shows that if $w_{1}, w_{2}, \ldots, w_{n}$ are $n$ distinct points in $\{|z|<1\}$, and if $b_{1}, b_{2}, \ldots, b_{n}$ are complex numbers, then the interpolation problem

$$
F\left(w_{j}\right)=b_{j}, \quad j=1,2, \ldots, n
$$

has a solution of the form $F=\lambda B$ where $\lambda$ is a constant, and $B$ is a Blaschke product of degree $\leqq n-1$. Furthermore, there is no trouble in interpolating a finite number of the derivatives of $F$ at the points $w_{j}$.

\section{REFERENCES}

(1) V. M. Adamyan, D. Z. Arov and M. G. Krein, Infinite Hankel matrices and generalized problems of Carathéodory, Fejer and F. Riesz, Functional Anal. Appl. 2 (1968), 1-14.

(2) L. CARLESON and S. JACOBs, Best uniform approximation by analytic functions, Ark. Mat. 10 (1972), 219-229. 
(3) T. W. Gamelin, J. Garnett, L. A. Rubel and A. L. Shields, On badly approximable functions, J. Approximation Theory, to appear.

(4) S. J. POREDA, A characterization of badly approximable functions, Trans. Amer. Math. Soc. 169 (1972), 249-256.

(5) D. Sarason, Algebras of functions on the unit circle, Bull. Amer. Math. Soc. 79 (1973), 286-299.

UNIVERSITY OF ILLINOIS AT

URBANA-CHAMPAIGN

UNIVERSITY OF MICHIGAN

E.M.S. $-20 / 2-1$ 